\title{
Interactions between Restorative Dentistry and Periodontics: Luting Post Nonmetallic (Part II)
}

\author{
${ }^{1}$ Matheus Coelho Bandéca, ${ }^{2}$ Shelon Cristina Souza Pinto, ${ }^{3}$ Mateus Rodrigues Tonetto, ${ }^{4}$ Fausto Frizzera \\ ${ }^{5}$ Kamila de Figueiredo Pereira, ${ }^{6}$ Alvaro Henrique Borges, ${ }^{7}$ José Roberto Cury Saad, ${ }^{8}$ Reidson Stanley Soares dos Santos
}

\begin{abstract}
Endodontically treated teeth have necessary use of intracanal posts. Due to the constant need and search for esthetic materials with properties similar to tooth structure remaining fiberglass posts esthetic came good these shortcomings and are increasingly used as a first option for prosthetic rehabilitation. In this case report is described step by step how to perform the cementation of a nonmetallic post and core construction for making a piece prosthetic onlay in a simplified way, by choosing the self-etching cementation technique.
\end{abstract}

Keywords: Restorative dentistry, Fiber post, Luting agents.

How to cite this article: Bandéca MC, Pinto SCS, Tonetto MR, Frizzera F, de Figueiredo Pereira K, Borges AH, Saad JRC, dos Santos RSS. Interactions between Restorative Dentistry and Periodontics: Luting Post Nonmetallic (Part II). World J Dent 2014;5(1):72-75.

Source of support: Nil

Conflict of interest: None

\section{INTRODUCTION}

Teeth with extensive coronal destruction and endodontically treated still a challenge for the dentist in their daily practice. ${ }^{1}$ The dental element, weakened due to caries, sobrepreparo, fracture, internal resorption or removal of unsatisfactory restorations, often needs, a retainer intraradical for your complete repair functional and esthetic. ${ }^{2}$

Formerly restorative dentistry used to cast metal cores, materials having elastic modulus with significant differences from the tooth structure, besides higher wear element already fragile, requiring more clinical sessions and having

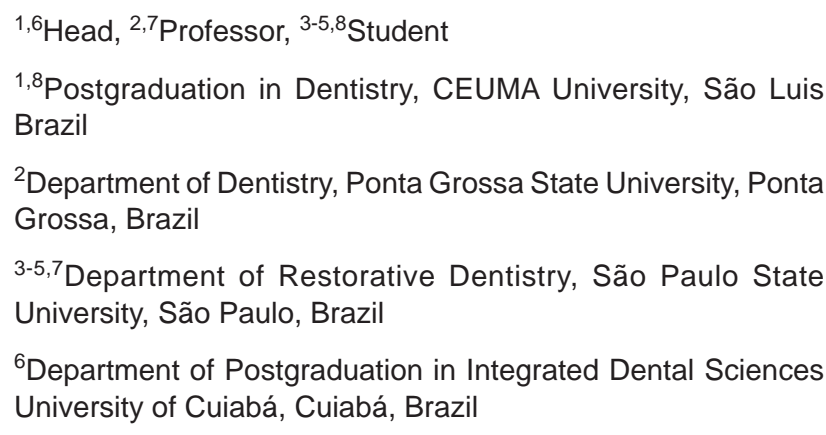

${ }^{6}$ Department of Postgraduation in Integrated Dental Sciences University of Cuiabá, Cuiabá, Brazil

Corresponding Author: Matheus Coelho Bandéca, Head Postgraduate Program in Dentistry, CEUMA University, Rua Josue Montello, n 01, Renascença, São Luis, MA 65075-120 Brazil, e-mail: matheus.bandeca@ceuma.br high cost. With the advancement of technology, the change inherent in the composition of the materials was gradually passing to biomimeticamente characteristics similar to dentin, assisting in the transfer of force equally throughout the tooth, resulting in lower rates of fracture and stress due to their modulus of elasticity equivalent to the dental. ${ }^{3}$ So with posts and esthetic evolution adhesive expanded the possibility of restorative techniques, fiberglass posts now used with the proposal to be biologically and esthetically favorable very advantageous by having technical adhesion and wear less simplified structure remaining. ${ }^{4}$

Given the above, the purpose of this article is to conduct a protocol through a clinical case of luting of esthetic restorative technique that is an option useful and simplified current treatment.

\section{CASE REPORT}

Female patient, 36 years old, attended the clinic Restorative Dentistry, Faculty of Dentistry of Araraquara, complaining of pain in element 16. After clinical and radiographic examination it was found that the restoration needed to be replaced and endodontic treatment performed. After removal of the restoration was necessary to perform a surgery to increase the clinical crown, it was invasion of the biologic width, reported and discussed in the first part of this article. With periodontal surgery and endodontic treatment made, steps described in this article.

\section{Root Canal Preparation}

It was decided during the planning of the case by the endodontic treatment of root canals element 16 and prosthetic restoration with a piece of ceramic onlay on IPS-Empress 2. Because it is a first molar palatal root was chosen to receive the post esthetic, to increase retention onlay, so after endodontic treatment completed this root had its apical preserved, leaving $4 \mathrm{~mm}$ of filling material inside. The removal procedure was completed with a milling cutter Largo \# 2 regulate the root canal and remove any endodontic material of dentin walls (Fig. 1).

\section{Selection Post}

According to the size of the root canal, the fiber post (FGM, Joinville, SC, Brazil), with a double taper, was chosen and verified by radiographic examination compatibility (Figs 2 and 3). 


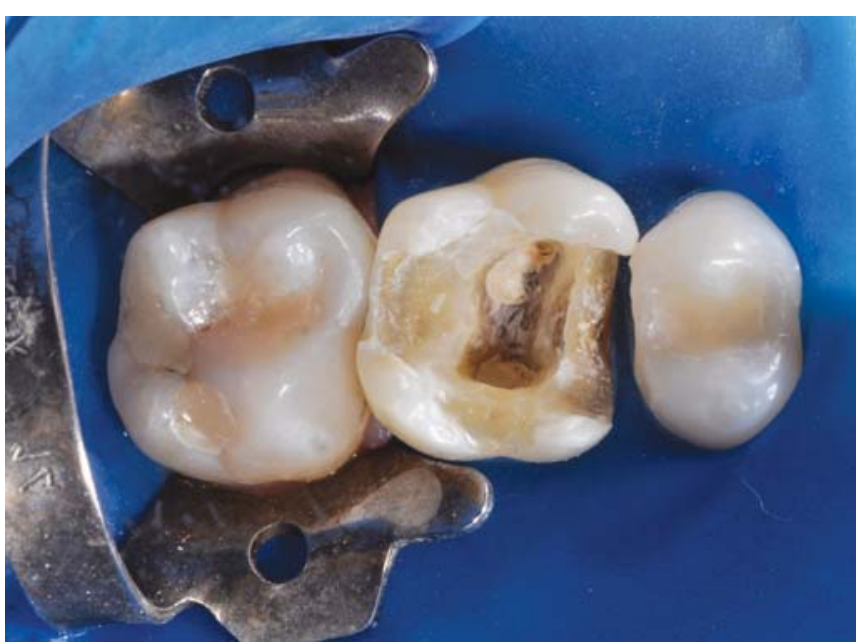

Fig. 1: Occlusal view of the canals after endodontic treatment

\section{Luting}

For the adhesive cementation procedure was made absolute isolation of the operative field. The cement was chosen type self-adhesive resin-U200 (3M ESPE)—simplified application without pretreatment of dentin. The surface of the glass fiber post was etched with 37\% phosphoric acid for 60 seconds (Figs 4A to C), rinsed with water spray for 30 seconds and dried (Fig. 5). The cement was inserted into the root canal along with the esthetic post (Figs 6 and 7), the whole was kept in position and removing excess was only after 5 seconds of light curing, and then the polymerization was terminated.

\section{Making the Core Filling}

The core filling was done following the protocol adhesive with phosphoric acid $37 \%$ of the remaining tooth and post for 15 seconds on dentin and enamel in 30 seconds. After application of the adhesive system Adper Scotchbond (3M ESPE) filling the core was built with composite microhybrid Filtek Z250 (3M ESPE), in preparation for onlay format (Figs 8 to 13).

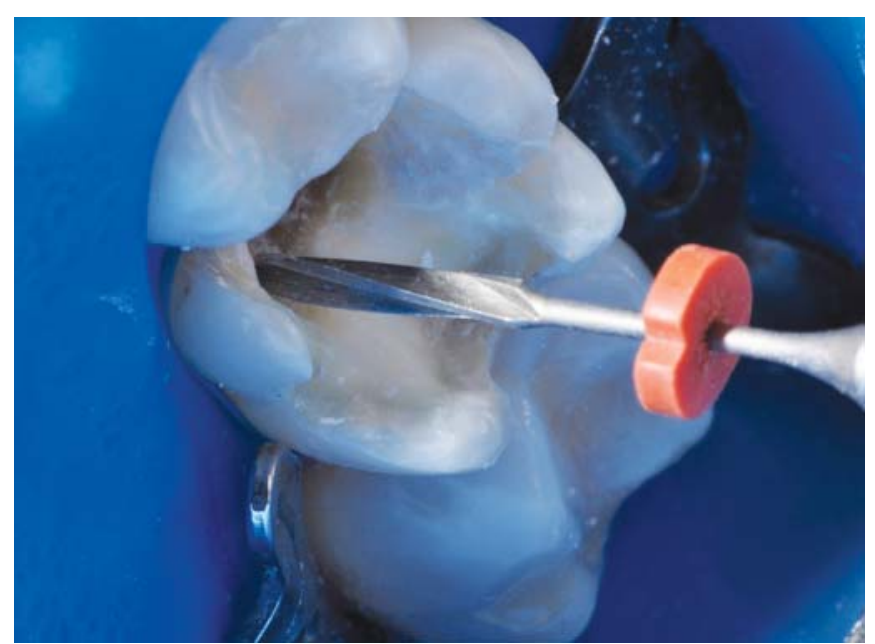

Fig. 2: The Largo was used to regulate the root canal and remove endodontic material of dentin walls

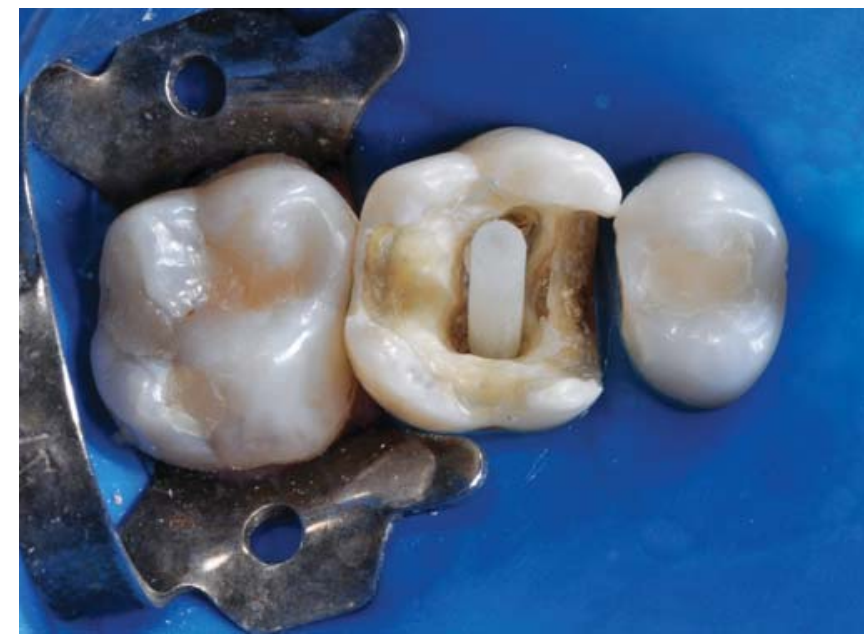

Fig. 3: The fiber post was checked inside the root canal preparation

\section{DISCUSSION}

The fiberglass posts helped create a more favorable biomechanical model of the dental structure. This concept came to aggregate more technical advantage of the restorative dentistry, where complex post/luting agent/tooth became more homogeneous, with physical properties similar
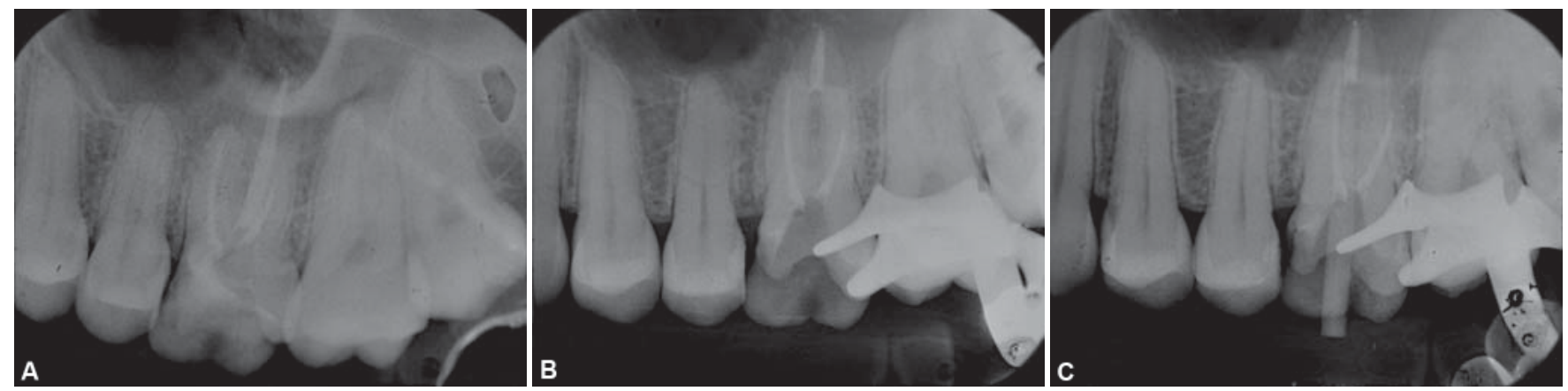

Figs 4A to C: Radiographs were taken to place the post: (A) initial radiograph was made to verify the length of root canal that will receive the post, $(B)$ filling material was removed and checked by radiography, $(C)$ radiograph to verification of the post within the channel 


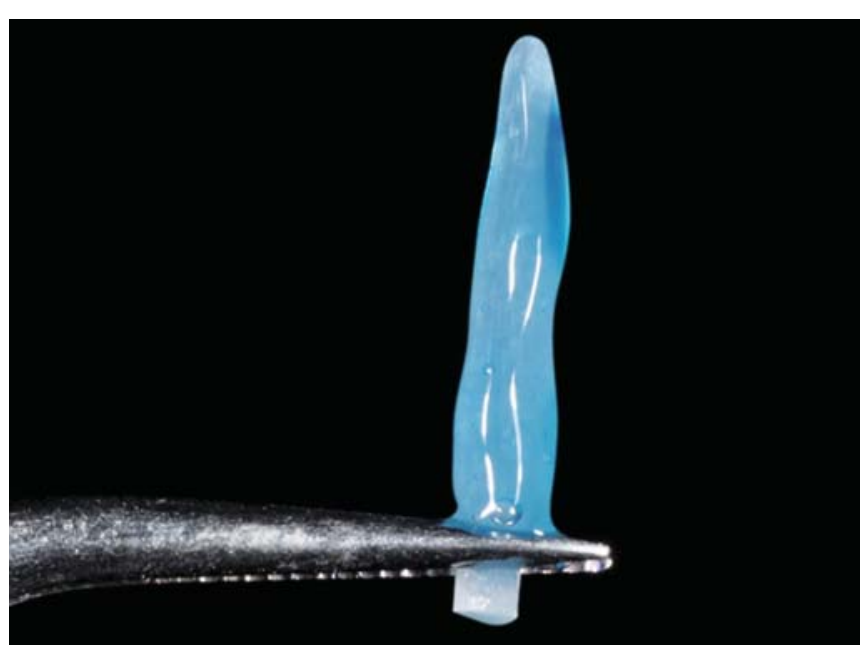

Fig. 5: The fiber post was etched with $37 \%$ phosphoric acid for 60 seconds

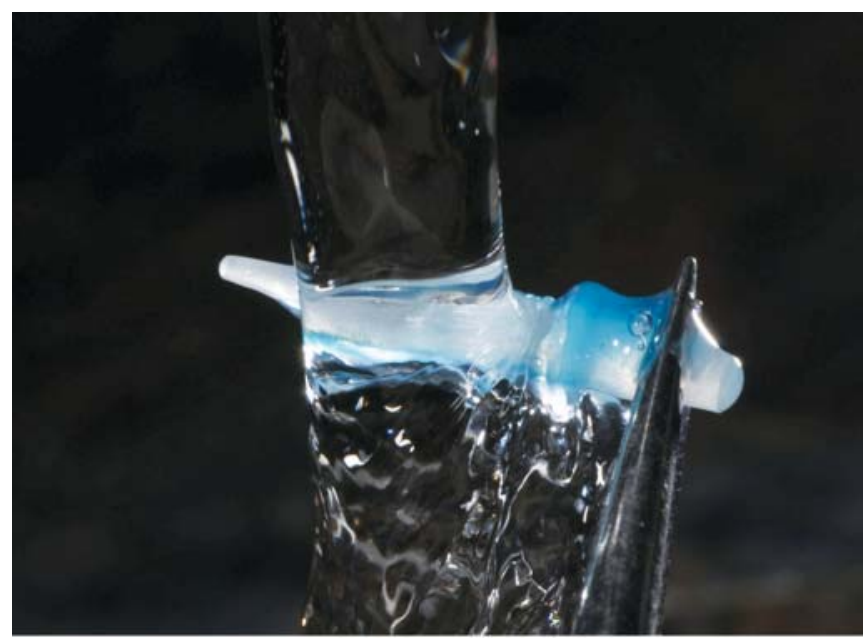

Fig. 6: The fiber post was rinsed with water spray for 30 seconds

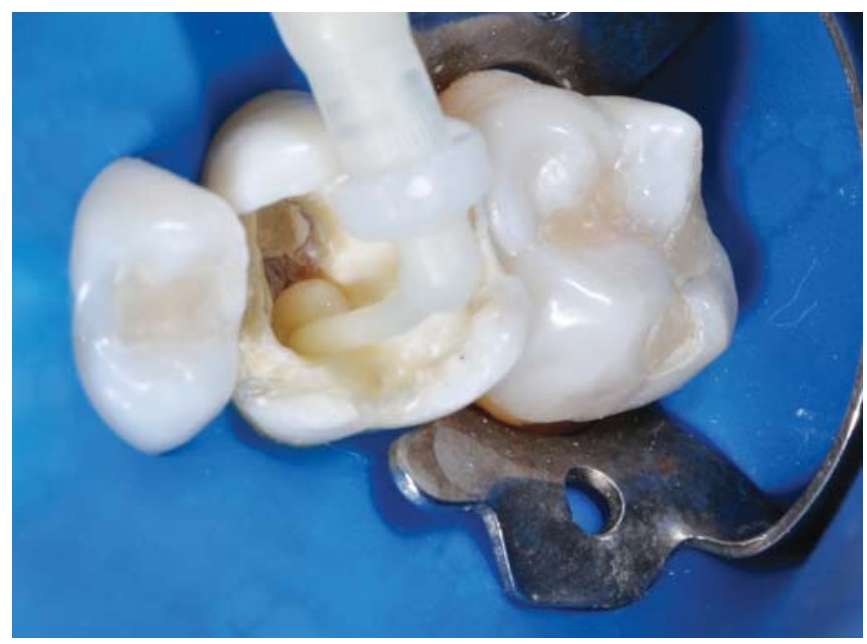

Fig. 7: The cement was inserted inside the root canal preparation

to dentin. Thus, the transfer of stress caused by occlusal load or even trauma, adequate remaining tooth enabled the lower rate of root fracture. ${ }^{2,5}$

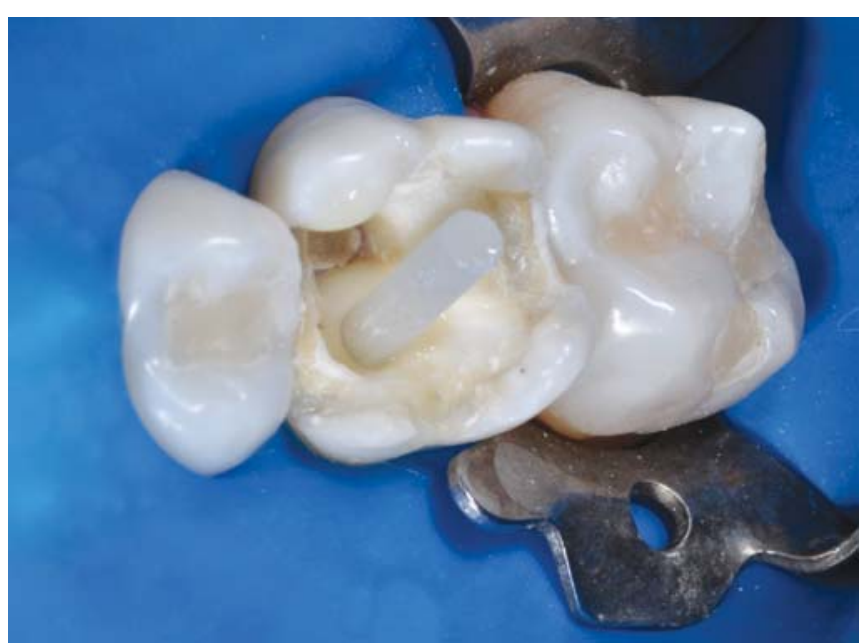

Fig. 8: The fiber post was inserted inside the root canal

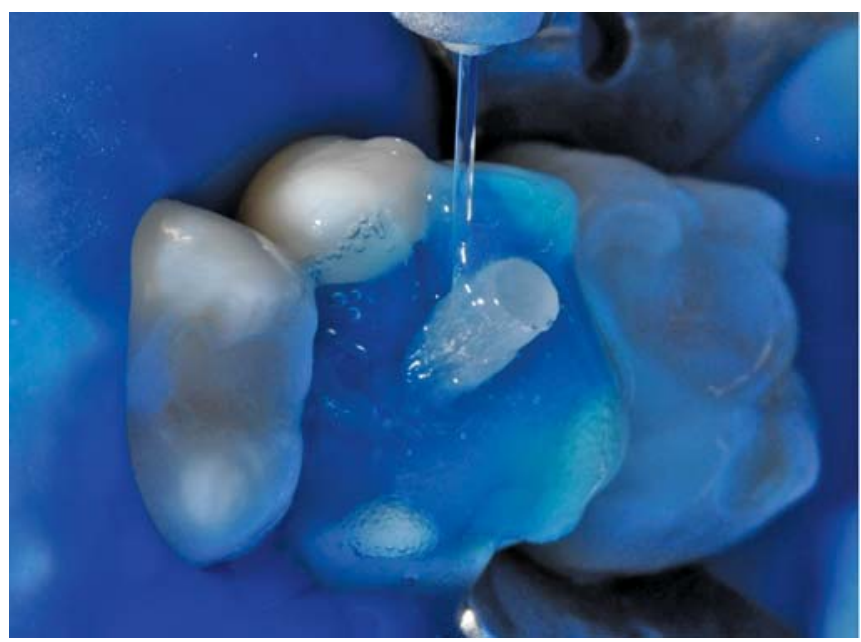

Fig. 9: The phosphoric acid $37 \%$ was inserted in remaining tooth for 15 seconds on dentin and 30 seconds in enamel. After, it was removed with water

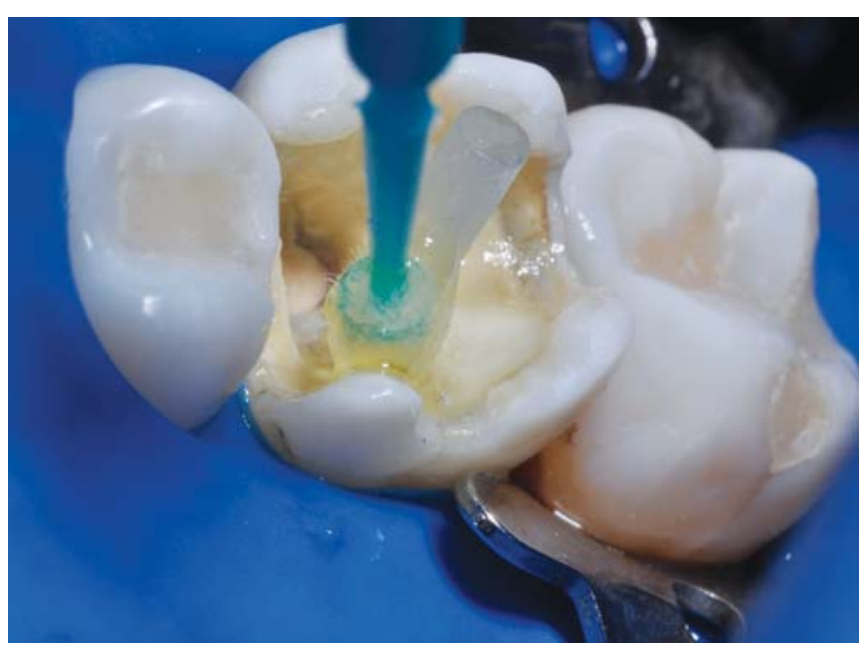

Fig. 10: The adhesive system Adper Scotchbond was used before filling the core

However we should pay more attention to the compatibility between the components from the adhesive cementation technique, where differences can occur between 


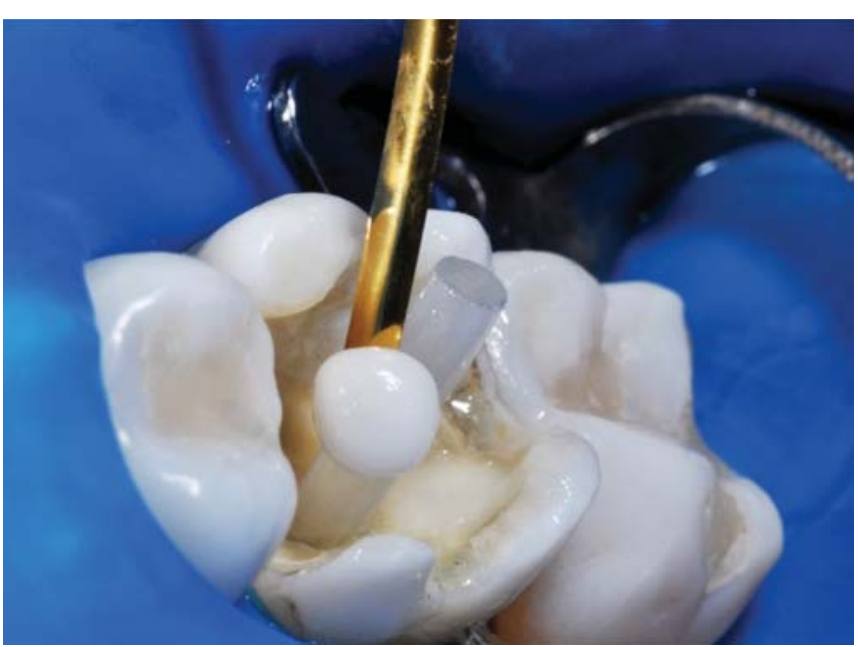

Fig. 11: The composite resin was applied in preparation for onlay format

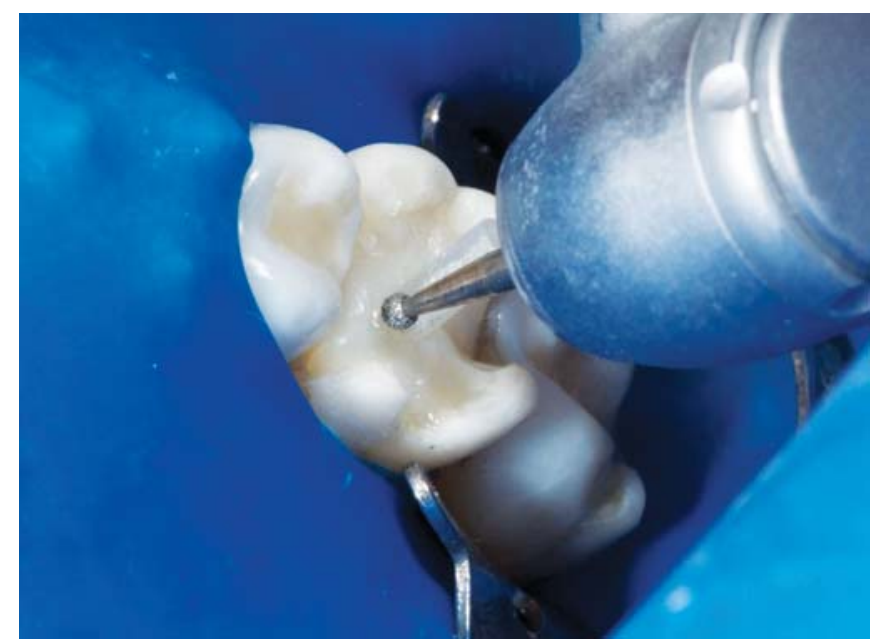

Fig. 12: Remainder of the post was removed with a spherical bur. This procedure was made only after filling the core, because it prevents fracture of the cement by vibration of bur

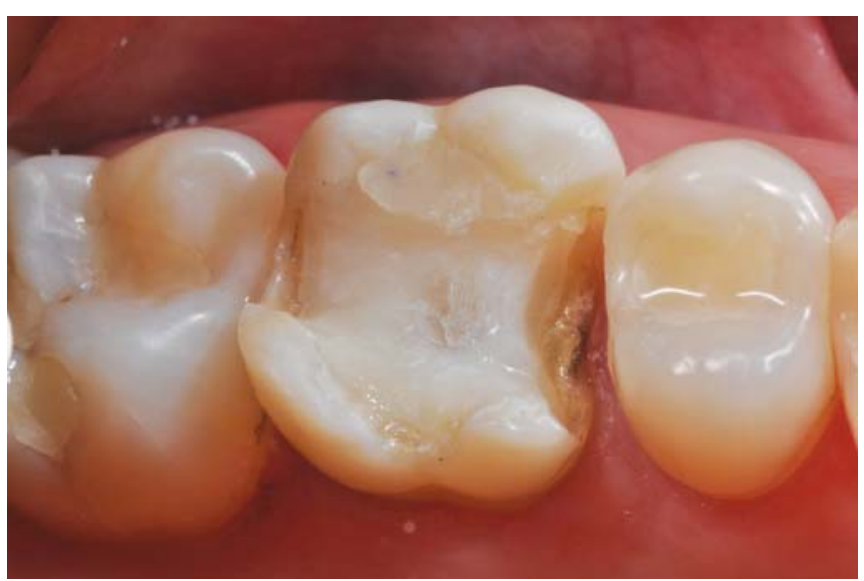

Fig. 13: Final view of the filling core with post

the adhesive system and luting agent. ${ }^{6,7}$ The presence of acidic monomers in some conventional adhesive systems single bottle has the capability of negative interaction with chemically activated resins, observed in duals cements. The simplicity of the technique using self-etching resin cement requires no adhesive system and etching, the fact that from the choice of this procedure in this clinical case., ${ }^{9,10}$

A study by Ferrari ${ }^{8}$ found adherence of fiber glass in a longitudinal where it was observed that only $3.2 \%$ of the failures were adhesive technique. Thus the bond strength observed in the literature confirms the efficacy of the technique and its indication when you need to opt for esthetics, along with the function.

Given the current need for esthetics alongside evolution of adhesive systems and luting agents posts esthetic took their place in daily practice.

\section{CONCLUSION}

Through this paper we can show that the use of post esthetic technique has advantages of simplicity with materials having modulus of elasticity close to dentin.

\section{REFERENCES}

1. Mosharraf R, Ranjbarian P. Effects of post surface conditioning before silanization on bond strength between fiber post and resin cement. J Adv Prosthodont 2013;5:126-132.

2. Ferrari M, Vichi A, García-Godoy F. Clinical evaluation of fiberreinforced epoxy resin posts and cast post and core. Am J Dent 2000;13:15B-18B.

3. Eskitascioglu G, Belli S, Kalkan M. Evaluation of two post core system using two different methods (fracture strength test and a finite elemental stress analysis). J Endod 2002;28:629-633.

4. Monticelli F, Goracci C, Grandini, S, Garcia-Godoy F, Ferrari M. Scanning electron microscopic evaluation of fiber post-resin core units built up with different resin composites. Am J Dent 2005;18:61-65.

5. Lassila LP, Tanner J, Le Bell AM, Narva K, Vallittu PK. Flexural properties of fiber reinforced root canal posts. Dent Mater J 2004;20:29-36.

6. Suh BI, Feng L, Pashley DH, Tay FR. Factors contributing to the incompatibility between simplified-step adhesives and chemically-cured or dual-cured composites. Part III. Effect of acidic resin monomers. Adhes Dent 2003;5:267-282.

7. Egilmez F, Ergun G, Cekic-Nagas I, Vallittu PK, Lassila LV. Influence of cement thickness on the bond strength of toothcolored posts to root dentin after thermal cycling. Acta Odontol Scand 2013;71:175-182.

8. Ferrari M, Vich A, Mannocci F, Mason PN. Retrospective study of the clinical performance of fiber post. Am J Dent 2000;13:9-13.

9. Calixto LR, Bandéca MC, Clavijo VGR, Andrade MF, Vaz LG, Campos EA. Effect of resin cement system and root region on the push-out bond strength of a translucent fiber post. Operative Dentistry 2012;37:80-86.

10. Clavijo VRG, Bandéca MC, Calixto LR, Nadalin MR, Saade EG, Oliveira-Junior OB, Andrade MF. Factors affecting on bond strength of glass fiber post cemented with different resin cements to root canal. Laser Physics 2009;19:1920-1924. 